\title{
Effect of pre-game photobiomodulation on muscle recovery in professional soccer players: study protocol for a randomized, cross-over, sham-controlled, triple-blind, clinical trial.
}

\author{
Igor Phillip dos Santos Glória', Ernesto Cesar Pinto Leal Junior'1, Fabiano Politti', Carolina Marciela Herpich'1, \\ Denis Smith1', Leandro Silva de Carvalho', Cid André Fidelis de Paula Gomes², Tabajara de Oliveira Gonzalez², \\ Daniela Aparecida Biasotto-Gonzalez',2
}

\begin{abstract}
Background: Photobiomodulation with low-level laser therapy (LLLT) has been widely used in clinical practice for diverse purposes, such as modulation of the inflammatory process, acceleration of the tissue repair process, pain relief and the enhancement of post-exercise recovery. Studies have demonstrated a beneficial interaction between photobiomodulation and the production of creatine kinase, with a reduction in the release of this marker of muscle damage when laser and/or LEDs is administered prior to high-intensity physical activity. Objective: The aim of the proposed study is to determine the influence of pre-exercise phototherapy on post-exercise muscle recovery. Methods: A randomized, cross-over, sham-controlled, double-blind, clinical trial is proposed. The participants will be healthy professional soccer players aged 15 to 20 years from the same team with a body mass index within the ideal range $\left(20\right.$ to $\left.25 \mathrm{~kg} / \mathrm{m}^{2}\right)$ and no history of lower limb musculoskeletal injuries or surgery or back surgery in the previous six months. The athletes will be allocated to two groups based on the previously calculated sample size and will be blinded to allocation. Creatine kinase will be measured and the subjective perception of fatigue will be determined for each participant. The volunteers will then be randomly allocated to Group A, which will receive active phototherapy, and Group B, which will receive sham phototherapy. The athletes will undergo reevaluations immediately after as well as 48 hours after a football match. The data will be submitted to statistical analysis and the level of significance will be set to $5 \%$. This study is registered with Clinical Trials under protocol number: NCT03416998.
\end{abstract}

Key words: Phototherapy; Photobiomodulation; Creatine kinase; Soccer; LLLT; Muscle Damage; Skeletal Muscle Recovery; Lasers.

\section{INTRODUCTION}

The positive effects of photobiomodulation (PBMT), with low-level laser therapy (LLLT) and/or light-emitting diode therapy (LEDT) have been studied over the years. The photochemical effect on cells through the absorption of light by photoreceptors ${ }^{(1,2)}$ is denominated photobiostimulation or photobiomodulation ${ }^{(3)}$. The physiological theory for such benefits regards the interaction between the penetrating light and biological tissue. PBMT is believed to activate cytochrome c oxidase (COX), which is the main chromophore in cells capable of absorbing specific wavelengths of light ${ }^{(4,5)}$. Thus, PBMT has been widely used for different types of medical treatment, especially when cell stimulation is required. PBMT is a therapeutic tool that consists of electromagnetic waves and the media used for its production, with low-intensity energy and wavelengths capable of penetrating tissues, thereby influencing the synthesis, release and metabolism of numerous signaling substances involved in analgesia ${ }^{(6,7)}$.
Since the publication of the first randomized clinical trial to investigate the effects of PBMT on disorders of the musculoskeletal system in patients with rheumatoid arthritis in the 1980 's ${ }^{(8)}$, the effects of this therapeutic modality on adverse health conditions, muscle recovery and improved muscle performance have been studied. Hypotheses have been put forth regarding the analgesic action of PBMT, such as the modulation of inflammatory processes ${ }^{(9,10)}$, modulation of the excitation and nerve conduction of peripheral neurons ${ }^{(11)}$, the release of endogenous opioids ${ }^{(12)}$ and an increase in the synthesis of serotonin ${ }^{(13)}$. PBMT is also reported to promote vasodilatation and increase blood flow ${ }^{(14,15)}$.

Muscle damage stemming from physical activity in which the muscle is submitted to situations of fatigue is characterized by structural abnormalities, including sarcomere disorders and membrane injuries, resulting in the loss of soluble enzymes, such as creatine kinase (CK), which is often found in

*Corresponding author: Igor Phillip dos Santos Glória - Universidade Nove de Julho (UNINOVE). Address: Av. Dr. Adolfo Pinto,109, Água Branca, 05001-100. São Paulo, SP, Brazil. E-mail: igorgloria@yahoo.com.br - Phone: +55 11 99546-6201

1 Postgraduate Program in Rehabilitation Sciences, Universidade Nove de Julho (UNINOVE). Address: Av. Dr. Adolfo Pinto, 109, Água Branca, 05001-100. São Paulo, SP, Brazil. Full list of author information is available at the end of the article.

Funding: Funding for this study was provided by the Brazilian Coordination for the Improvement of Higher Education Personnel (CAPES).

Submission date 12 August 2018 ; Acceptance date 23 September 2018 ; Publication date 11 November 2019 
high levels in the blood stream after the practice of physical exercise $^{(16)}$. Studies have demonstrated a beneficial interaction between PBMT and the production of $\mathrm{CK}$, with a reduction in the release of this marker of muscle damage when laser is administered prior to high-intensity physical activity ${ }^{(17)}$. This positive response is explained by the theory that PBMT is capable of improving the function of cellular mitochondria(18). PBMT promotes an interaction between light and the target tissue through the stimulation of $\mathrm{COX}$, which is a mitochondrial enzyme that plays an important role in the electron transport chain and consequently promotes an improvement in cell respiration and the production of energy in the form of adenosine triphosphate ${ }^{(4,5,19)}$.

There is considerable discussion on the best time to administer PBMT (before or after the practice of physical activity) for the enhancement of the muscle recovery process. In a recent systematic review and meta-analysis, Leal-Junior et al. ${ }^{(20)}$ analyzed 13 articles and concluded that PBMT with LLLT and LEDT is more beneficial when administered prior to physical activity, as it preserves the muscle tissue and accelerates post-exercise recovery time. Ferraresi et al. (21) investigated the effects of PBMT with LEDT on the prevention of muscle damage through an analysis of CK in professional volleyball players. The authors tested four different energy doses on four groups of athletes (one of which was sham therapy) and found positive effects when the total energy per muscle group was 210 and $315 \mathrm{~J}$ (exposure time of 40 and 60 s, respectively). It should be stressed that this was not a cross-over study and the volunteers who received sham therapy were not subsequently submitted to PBMT with LEDT. Leal-Junior et al. ${ }^{(22)}$ evaluated the effects of pre-exercise PBMT on muscle damage markers [blood concentrations of CK (measured before activity and 3 min after activity) and lactate (measured before activity as well as 3, 10 and 15 min after activity)] in a cross-over study and concluded that pre-exercise phototherapy was capable of reducing muscle damage.

Soccer (European football) is the most popular sport, with 200,000 professional players and 240 million amateur players throughout the world, approximately $80 \%$ of whom are men $^{(23)}$. Due to such popularity, the sport has been the topic of intensive research in the last ten years. The competitive demands of soccer tax the musculoskeletal, nervous, metabolic and immune systems to such a point that post-exercise recovery strategies are needed to prepare athletes for the next match ${ }^{(24)}$. In Brazil, soccer players are more prone to injury due to the intensive training and large number of games. The incidence of injury in soccer is estimated to be 10 to 15 injuries per every 1000 hours of practice. However, this figure varies considerably among studies, depending on the definition of injury employed ${ }^{(25)}$. A soccer match is characterized by periods of high-intensity exercise intercalated with periods of low intensity. The physiological demands of the sport require a high level of physical training involving aerobic and anaerobic power, muscle strength and agility ${ }^{(26,27)}$. During a soccer season, player performance is not determined only by adequate physical fitness, but also by the recovery capacity following the exposure to various stressful stimuli during matches and daily practice ${ }^{(26)}$. Studies have demonstrated an increase in the muscle damage marker $\mathrm{CK}$ after the practice of soccer ${ }^{(28-31)}$.

\section{HYPOTHESIS}

The hypothesis of the proposed study is that pre-exercise PBMT is capable of enhancing post-exercise muscle recovery in professional soccer players.

Primary outcome: Muscle Recovery post-exercise

Secondary Outcome: Sports Performance

\section{OBJECTIVES}

\section{General objective}

The general objective of the proposed study is to determine the influence of pre-exercise PBMT on muscle recovery.

\section{Specific objectives}

- Determine the influence of pre-exercise PBMT on the physiological marker creatine kinase (CK);

- Determine the influence of pre-exercise PBMT on a subjective perception of fatigue;

- Analyze and compare the influence of pre-exercise PBMT at different recovery times (immediately after exercise and $48 \mathrm{~h}$ after exercise).

\section{METHODS}

\section{Design}

A randomized, cross-over, sham-controlled, double-blind, clinical trial on the influence of pre-exercise PBMT on the post-exercise recovery of professional soccer players from the same team

\section{Approval}

This project has been submitted for approval from the Human Research Ethics Committee of University Nove de Julho (São Paulo, Brazil) under protocol number 55847716.6.0000.5511.

\section{Sample}

A pilot study will be first conducted to offer a basis for the calculation of the sample size. The study will involve the participation of male professional soccer players aged 15 to 20 years selected randomly from the same team. All participants must meet the inclusion criteria and agree 
to take part in the study. The study will be conducted in compliance with the norms that govern research involving human subject stipulated in Resolution $n^{\circ} 466 / 12$ of the Brazilian National Board of Health. All participants and legal guardians of minors who will be evaluated will receive clarifications regarding the objectives of the study and will be asked to sign a statement of informed consent.

\section{Inclusion criteria}

Healthy professional soccer players aged 15 to 20 years from the same team with a body mass index in the ideal range ( 20 to $25 \mathrm{~kg} / \mathrm{m}^{2}$ ) will be included in the study.

\section{Exclusion criteria}

A history of musculoskeletal injuries and/or surgeries in the lower limbs or back surgery in the previous six months

\section{Randomization and blinding}

The athletes will be allocated to two groups based on the previously determined sample calculation and will be blinded to allocation. Randomization will be performed using a statistical program found at the electronic address www. randomization.com. 1:1 randomization will be conducted in blocks, with permutation and altering the size of the blocks. Randomization and allocation concealment will be performed by Researcher 1, who will also be in charge of programming the PBMT device based on the results of the randomization process. A second researcher will calibrate the PBMT device and leave it switched on with the correct dosage or in sham mode and will not be aware of which therapy is being administered to each athlete. A third researcher will administer the PBMT without being aware of whether the athlete is receiving active or sham irradiation. The device emits the same sounds and illumination when set to the correct energy dose or in sham mode. The statistician will also be blinded to the allocation of the participants until the end of the analyses. Using this procedure, each athlete will have the same probability of being allocated to one of the two treatment groups. Moreover, the blinding of the evaluator and participants will be maintained until the end of the study and tabulation of the data.

\section{Procedures}

As the proposed study is a randomized clinical trial, the recommendations of the CONSORT (Consolidated Standards of Reporting Trials) statement will be followed ${ }^{(32)}$. First, CK will be measured and the Scale of Perceived Exertion will be administered. The athletes will then be randomly allocated to Group A (active p PBMT) and Group B (sham PBMT). The volunteers will participate normally in an official soccer match. CK will be measured and the scale of will be administered a second time at the end of the match and again $48 \mathrm{~h}$ after the match.

\section{Blood collection for analysis of creatine kinase}

Blood will be collected for the analysis of CK by a professional nurse with five years of experience. The skin will be cleaned with $70 \%$ alcohol, the tip of the index finger will be punctured automatically with the needle pen and $32 \mu \mathrm{L}$ of capillary blood will be drained into a heparinized capillary tube (Cat $n=955053202$ Reflotron $^{\circledR}$ ) and the blood will be pipetted to a CK reactive strip (Cat no 1126695 Reflotron ${ }^{\circledR}$ ), which will be placed into the Reflotron Analyser ${ }^{\circledR}$, (Boehringer Mannheim) for analysis.

\section{Subjective perception of fatigue (SPF)}

The subjective perception of fatigue (SPF) is a scale that was adapted based on the Borg scale, which was originally developed in a variation of 6 to 20 and can be used as a parameter determining the intensity of the activity(33). It takes into account during its measurement, physical and psychological factors, and these factors together determine the level of physical fatigue after a certain activity. To evaluate the subjective perception of fatigue, we adapted the scale to a graduation from 0 to 10 , because it has a better understanding by the athletes, and the answer 0 indicates totally rested and very tired ${ }^{(34)}$. Each participant will be given an explanation of each item before the administration of the scale.

\section{Photobiomodulation}

Active (Group A) or sham (Group B) PBMT will be administered 30 minutes prior to the soccer match as well as $48 \mathrm{~h}$ after the match in direct contact with the skin with light pressure at predetermined sites: nine on the knee extensor muscles (Figure 1), six on the knee flexor muscles (Figure 2) and two on the gastrocnemius muscle (Figure 3 ) on both lower limbs.

PBMT will be performed using clusters of 12 diodes (Table 1 ); four $905 \mathrm{~nm}$ diodes (mean power of $0.3125 \mathrm{~mW}$ and peak power of $12.5 \mathrm{~W}$ for each diode; $250 \mathrm{~Hz}$ ), four $875 \mathrm{~nm}$ diodes (mean power of $17.5 \mathrm{~mW}$ for each diode) and four $640 \mathrm{~nm}$ diodes (mean power of $15 \mathrm{~mW}$ for each diode). The device manufactured by Multi Radiance Medical ${ }^{\circledR}$ (Solon, OH, USA) will be used due to its high quality, ample use in clinical practice and due to the fact that no national companies manufacture diode clusters with the characteristics required for the proposed project. Considering the extensive irradiation area employed in the proposed study, the use of clusters will be fundamental to the application of phototherapy. The device employed has a circular cluster with a total area of $20 \mathrm{~cm}^{2}$. The dose used for active PBMT will be $30 \mathrm{~J}$ per area (228 seconds of exposure time in each area): $510 \mathrm{~J}$ of irradiated energy per lower limb and $1020 \mathrm{~J}$ of total irradiated energy. The dose to be employed at each site has previously been established and tested by our research group with the same PBMT equipment ${ }^{(36)}$. For sham treatment, the same procedures and treatment times will be employed, but the equipment will be set in placebo mode. Only the researcher in charge of programming the device will have knowledge regarding which treatment is being used. However, 


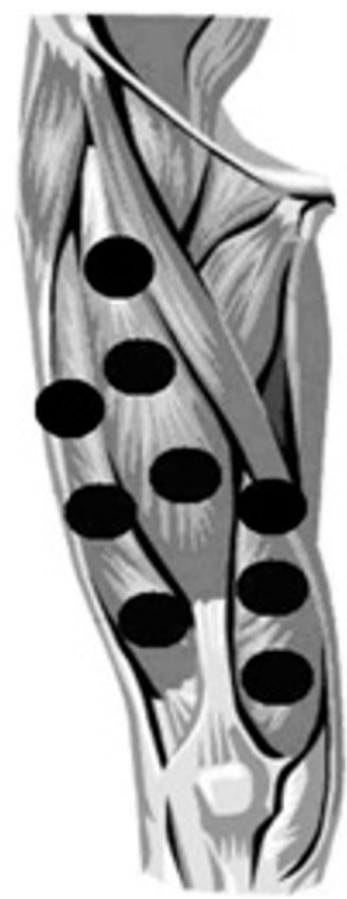

Figure 1: Photobiomodulation application sites in anterior region of lower limbs

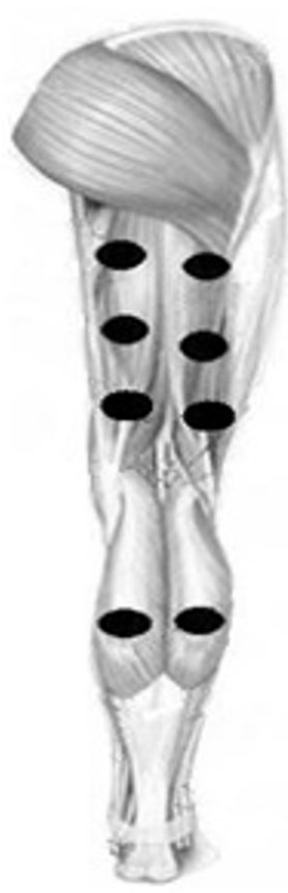

Figure 2: Photobiomodulation application sites in posterior region of lower limbs

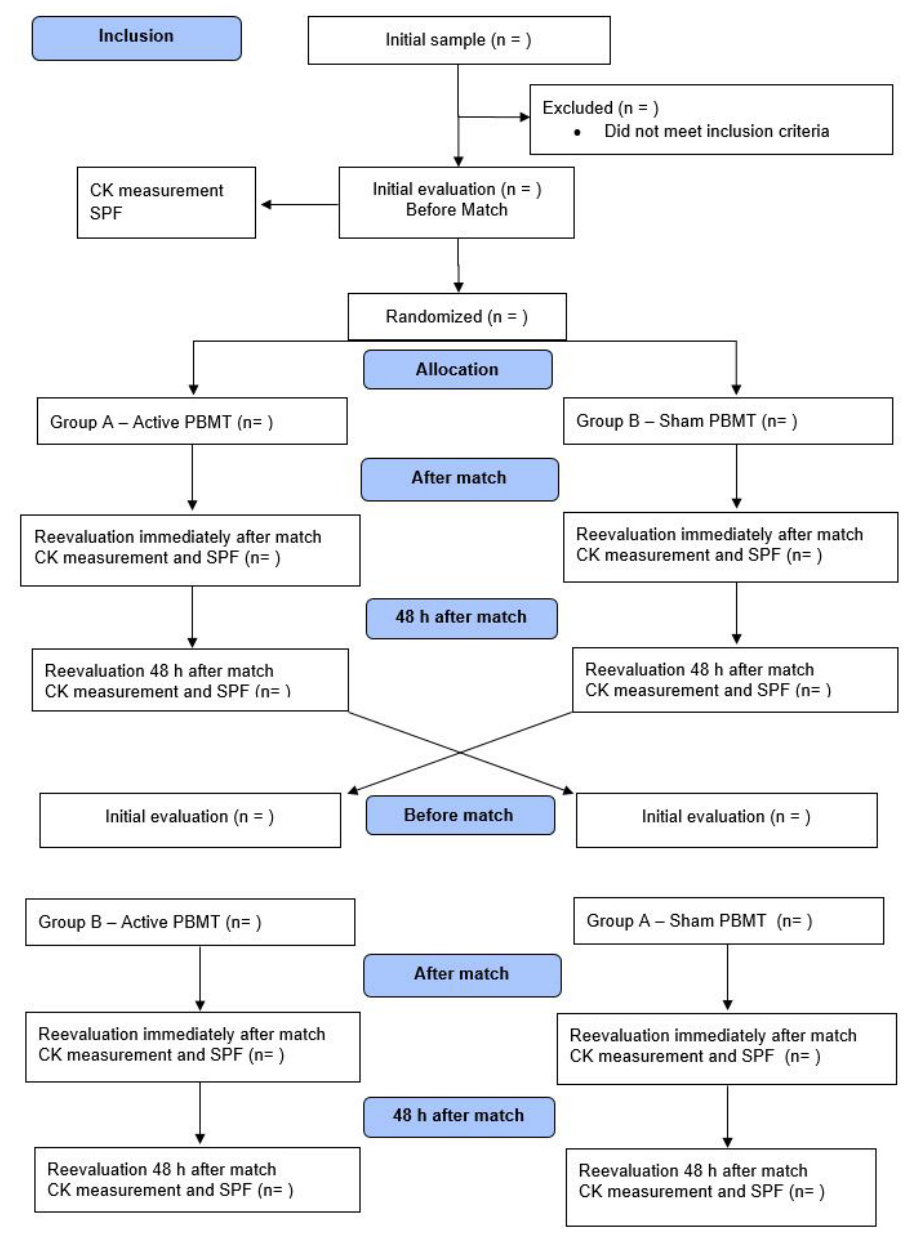

Figure 3: Flowchart (CONSORT, 2010) 
Table 1. Photobiomodulation Parameters

Number of clusters
Number of diodes
Number of irradiation points per
muscle

Number of lasers

Wavelength (nm)

Frequency $(\mathrm{Hz})$

Mean optical output (mW)

Peak power (W)

Dose $(J)$ of each diode $(228 \mathrm{~s})$

Spot size of laser $\left(\mathrm{cm}^{2}\right)$

Number of LEDs

Wavelength $(\mathrm{nm})$

Frequency $(\mathrm{Hz})$

Mean optical output ( $\mathrm{mW}$ )

Dose $(J)$ of each diode (228 s)

Spot size of LED $\left(\mathrm{cm}^{2}\right)$

Number of LEDs

Wavelength $(\mathrm{nm})$

Frequency $(\mathrm{Hz})$

Mean optical output ( $\mathrm{mW}$ )

Dose $(\mathrm{J})$ of each diode (228 s)

Spot size of LED $\left(\mathrm{cm}^{2}\right)$

Total area of cluster

Exposure time

Energy delivered per cluster

Energy delivered per lower limb

Total energy delivered

Application mode

\section{7 (9 for quadriceps, 6 for \\ hamstrings and 2 for triceps surae) \\ 12 per cluster (4 at $905 \mathrm{~nm}, 4$ at $875 \mathrm{~nm}$ and 4 at $640 \mathrm{~nm}$ ) \\ 9 for quadriceps, 6 for hamstrings and 2 for triceps surae}

4 super-pulsed infrared
905
250
0.3125
12.5
0.07125
0.44
4 red
640
2
15
3.42
0.9

4 infrared
875
16
17.5
3.99
0.9

$20 \mathrm{~cm}^{2}$

$228 \mathrm{~s}$

$30 \mathrm{~J}$

$510 \mathrm{~J}$

$1020 \mathrm{~J}$

Cluster applied in stationary manner in contact with skin at 90ㅇ angle with light pressure

the programmer will not participate in the execution of the treatment, evaluations or data analysis.

\section{Subjective perception of fatigue}

0 - Rested

1 -

2 - Slightly tired

3 -

4-A little tired

$5-$

6 - Tired

$7-$

8 - Very tired

$9-$

10 - Exhausted

\section{DISCUSSION}

The proposed study will evaluate the effects of pre-exercise PBMT on muscle recovery in professional soccer players through an analysis of the enzyme creatine kinase (CK) measured prior to physical activity and immediately after as well as 24 and 48 hours after a soccer match. PBMT is a therapeutic resource that has been used to enhance muscle recovery in clinical trials with good results demonstrated by changes in markers of muscle damage, especially $\mathrm{CK}^{(35,36)}$. In a study conducted in a controlled environment, a beneficial interaction between PBMT and CK production was found, with a reduction in the release of this marker of muscle damage when PBMT was administered prior to high-intensity physical activity ${ }^{(22)}$. This positive response is explained by the theory that PBMT is capable of improving the function of cellular mitochondria(18). In a recent systematic review and meta-analysis, Leal-Junior et al. ${ }^{(20)}$ analyzed 13 articles and concluded that PBMT with LLLT and LEDT is more beneficial when administered prior to physical activity, as it preserves the muscle tissue and accelerates post-exercise recovery time. Studies addressing the use of PBMT on post-exercise recovery have demonstrated good results in controlled environments. Such benefits underscore the need to investigate whether the same effects are achieved in clinical practice.

\section{Acknowledgments}

The authors are grateful for all the support given by Nove de Julho University as well as all researchers and volunteers who participated in the study.

\section{AUTHORS' CONTRIBUTIONS}

All cited authors participated in these steps. IPSG: conception and design, data collection and analysis, manuscript writing and final approval of the manuscript. ECPLJ: conception and design, financial support, manuscript writing, final approval of manuscript. FP: data collection and analysis, critical revision and final approval of the manuscript. $\mathrm{CMH}$ : data collection and analysis, critical revision and final approval of the manuscript. CAFPG: data collection and analysis, critical revision and final approval of the manuscript. TOG: data collection and analysis, critical revision and final approval of the manuscript. DABG: conception and design, financial support, manuscript writing, final approval of manuscript.

\section{COMPETING INTERESTS}

The authors have declared that no competing interests exist.

\section{AUTHORS DETAILS}

2 Department of Physical Therapy, Universidade Nove de Julho (UNINOVE), Av. Dr. Adolfo Pinto, 109, Água Branca, 05001-100. São Paulo, SP, Brazil. 


\section{REFERENCES}

1. Huang YY, Chen AC, Carroll JD, Hamblin MR. Biphasic dose response in low level light therapy. Dose Response 7:358-383. 2009.

2. Lin F, Josephs SF, Alexandrescu DT, Ramos F, Bogin V, Gammill V et al., Lasers, stem cells, and COPD. J Trans/ Med. 8:16. 2010.

3. Mester E, Szende B, Gärtner P. The effect of laser beams on the growth of hair in mice. Radiobiol Radiother. 9:621-626. 1968.

4. Karu T. Primary and secondary mechanisms of action of visible to near-IR radiation on cells. J Photochem Photobiol. 49:1-17. 1999.

5. Karu TI. Multiple roles of cytochrome c oxidase in mammalian cells under action of red and IR-A radiation. IUBMB Life. 62: 607-610. 2010.

6. Marini I, Gatto MR, Bonetti GA. Effects of superpulsed low-level laser therapy on temporomandibular joint pain. Clin J Pain. 26:611-616. 2010.

7. Andrade TNC, Frade JC. Estudo comparativo entre os efeitos de técnicas de terapia manuais isolados e associada a laserterapia de baixa potência sobre a dor em pacientes com disfunção temporomandibular. Rev Gauch Odontol. 56: 287-295. 2008.

8. Goldman JA, Chiapella J, Casey H, Bass N, Graham J, Mcclatchey W et al. Laser therapy of rheumatoid arthritis. Lasers Surg Med. 1:93-101. 1980.

9. Bjordal JM. Effect of $830 \mathrm{~nm}$ low-level laser therapy applied before highintensity exercises on skeletal muscle recovery in athletes. Lasers Med Sci. 24: 857-863. 2009.

10. Chow RT, Johnson MI, Lopes-Martins RA, Bjordal JM. Efficacy of low-level laser therapy in the management of neck pin: a systematic review metaanalysis of randomized placebo or active-treatment controlled trials. Lancet. 374: 897-908. 2009.

11. Hadian ME, Moghagdam B. The effects of low power laser on electrophysiological parameters of sural nerve in normal subjects: a comparison between 670 and 780nm wavelengths. Acta Med Iran. 41:138-42. 2003.

12. Hagiwara S, Iwasaka H, Okuda K, Noguchi T. GaAlAs $(830 \mathrm{~nm}$ ) low-level laser enhances peripheral endogenous opioid analgesia in rats. Lasers Surg Med. 39:797-802. 2007.

13. Ceylan Y, Hizmetli S, Slig Y. The effects of infrared laser and medical treatments on pain and serotonin degradation products in patients with myofascial pain syndrome. A controlled trial. Rheumatoll Int. 24: 260-3. 2004.

14. Ferreira MC, Brito VN, Gameiro J, Costa MRS, Vasconcellos ECS, Cruz-Hofling MAC. Effects of HeNe laser irradiation on experimental paracoccidioidomycotic lesions. J Photochem Photob B: Biol. 84:141-9. 2006.

15. Rodrigo SM, Cunha A, Pozza DH, Blaya DS, Moraes JF, Weber JBB, et al. Analysis of the systemic effect of red and infrared laser therapy on wound repair. Photomed Laser Surg. 27:929-35. 2009.

16. Reihmane D, Jurka A, Tretjakovs P, Dela F. Increase in IL-6, TNF- $\alpha$, and MMP-9, but not sICAM-1, concentrations depends on exercise duration. European J Appl Physiology. 113:851-858. 2013.

17. Leal-Junior ECP, Lopes-Martins RAB, Baroni BM, De Marchi T, Taufer D, Manfro DS et al. Effect of $830 \mathrm{~nm}$ low-level laser therapy applied before high-intensity exercises on skeletal muscle recovery in athletes. Lasers Med Sci. 24:857-863. 2009.

18. Xu X, Zhao X, Liu TC, Pan H. Low-intensity laser irradiation improves the mitochondrial dysfunction of $\mathrm{C} 2 \mathrm{C} 12$ induced by electrical stimulation. Photomed Laser Surg. 26:197-202. 2008.

19. Karu TI, Pyatibrat LV, Kolyakov SF, Afanasyeva NI. Absorption measurements of cell monolayers relevant to mechanisms of laser phototherapy: reduction or oxidation of cytochrome c oxidase under laser radiation at 632.8 nm. Photomed Laser Surg. 26:593-599. 2008.
20. Leal-Junior ECP, Vanin AA, Miranda EF, De Carvalho PTC, Dal Corso S, Bjordal JM. Effect of phototherapy (low-level laser therapy and lightemitting diode therapy) on exercise performance and markers of exercise recovery: a systematic review with meta-analysis. Lasers Med Sci. 30: 925-939. 2015

21. Ferraresi C, Dos Santos RV, Marques G, Zangrande M, Leonaldo R, Hamblin MR et al. Light-emitting diode therapy (LEDT) before matches prevents increase in creatine kinase with a light dose response in volleyball players. Lasers Med Sci. 30(4):1281-7. 2015.

22. Leal-Junior ECP, Lopes-Martins RAB, Baroni BM, Marchi T, Rossi RP, Grosselli D et al. Comparison Between Single-Diode Low-Level Laser Therapy (LLLT) and LED Multi-Diode (Cluster) Therapy (LEDT) Applications Before High-Intensity Exercise. Photomed Laser Surg.. 27:617-623. 2009.

23. Timpka T, Risto O, Bjormsjo M. Boys soccer league injuries: a communitybased study of time-loss from sports participation and long-term sequelae. Eur J Public Health. 18:19-24. 2008.

24. Reilly T, Ekblom B. The use of recovery methods post-exercise. J Sports Sci. 23: 619-27. 2005.

25. Paus V, Torrengo F, Del Compare P. Incidence of injuries in juvenile soccer players. Rev Asoc Argent Traumatol Deporte. 10(1):28-34. 2003.

26. Reilly T, Drust B, Clark N. Muscle fatigue during football match-play. Sports Medicine, 38(5), 357-367, 2008.

27. Svensson M, Drust B. Testing soccer players. J Sports Sci. 23:601-618. 2005.

28. Andersson H, Raastad T, Nilsson J, Paulsen G, Garthe I, Kadi F. Neuromuscular fatigue and recovery in elite female soccer: effects of active recovery. Med Sci Sports Exerc. 40:372-80. 2008.

29. Ascensão A, Rebelo A, Oliveira E, Marques F, Pereira L, Magalhães J. Biochemical impact of a soccer match - analysis of oxidative stress and muscle damage markers throughout recovery. Clin Biochem. 41:841-51. 2008.

30. Ispirlidis I, Fatouros IG, Jamurtas AZ, Nikolaidis MG, Michailidis I, Douroudos I et al. Time-course of Changes in Inflammatory and Performance Responses Following a Soccer Game. Clin J Sport Med. 18:423-31. 2008

31. Magalhães J, Rebelo A, Oliveira E, Silva JR, Marques F, Ascensão A. Impact of Loughborough Intermittent Shuttle Test versus soccer match on physiological, biochemical and neuromuscular parameters. Eur J Appl Physiol. 108:39-48. 2010.

32. Schulz KF, Altman DG, Mohe D. CONSORT 2010 Statement: updated guidelines for reporting parallel group randomised trials CONSORT Statement.

33. Borg G. Psychophysical bases of perceived exertion. Med Sci Sports Exerc. 14:377-381. 1982.

34. Laurent CM, Green JM, Bishop PA, Sjokvist J, Schumacker RE, Richardson MT et al. A practical approach to monitoring recovery: Development of a perceived recovery status scale. J Strength Cond Res. 25: 620-628. 2011.

35. Antonialli FC, De Marchi T, Tomazoni SS, Vanin AA, dos Santos Grandinetti $\mathrm{V}$, de Paiva PR et al. Phototherapy in skeletal muscle performance and recovery after exercise: effect of combination of super-pulsed laser and light-emitting diodes. Lasers Med Sci. 29: 1967-76. 2014.

36. De Paiva PRV, Shaiane Silva Tomazoni SS, Johnson DS, Vanin AA, Albuquerque-Pontes GM, Machado CSM et al. Photobiomodulation therapy (PBMT) and/or cryotherapy in skeletal muscle restitution, what is better? A randomized, double-blinded, placebo-controlled clinical trial. Lasers Med Sci, 31: 1925-1933. 2016. 